\title{
Introduction to the Special Section on USENIX ATC 2019
}

This special section of ACM Transactions on Storage (ATC) presents some of the highlights of the storage-related papers published in the USENIX Annual Technical Conference (ATC'19). The scope of ATC is broad. It covers all practical aspects related to systems software, and its goal is to improve and further the knowledge of computing systems of all scales, from small embedded devices to large data centers, while emphasizing implementations and experimental results.

Despite its relatively broad scope, ATC can be easily characterized as (also) a primary storage conference, because it attracts many high-quality storage-related submissions-nearly as many as FAST. In particular, out of the 356 uploaded ATC'19 submissions, 123 (35\%) addressed various storage-related aspects. Correspondingly, out of its 71 accepted papers, $28(39 \%)$ addressed storagerelated themes, constituting a significant part of the ATC'19 program. Following are the specific subtopic statistics associated with the storage-related ATC'19 submissions as indicated by authors (each individual submission may be associated with multiple subtopics):

Subtopic
Deduplication
Erasure coding
Compression
Non-volatile memory
Remote memory
Caching
Key-value stores
Distributed storage systems
Filesystems
Flash memory and SSDs
Storage (general)

$\begin{array}{cc}\text { Submitted } & \text { Accepted } \\ 5 & 2 \\ 5 & 0 \\ 7 & 1 \\ 8 & 1 \\ 11 & 2 \\ 15 & 2 \\ 21 & 5 \\ 28 & 6 \\ 28 & 12 \\ 37 & 11 \\ 67 & 16\end{array}$

Of the preceding subtopics, for this special section of ATC, we selected two high-quality papers. Both include some additional material, which has been reviewed (in fast-track mode) by two of their original ATC'19 reviewers. The first paper is “The Reliability of Modern File systems in the Face of SSD Errors" by Shehbaz Jaffer, Stathis Maneas, Andy Hwang, and Bianca Schroeder, which studies errors and failures that affect the reliability of filesystems that are optimized for flash and are mounted on SSDs. The second paper is "Everyone Loves File: Oracle File Storage Service" by Bradley Kuszmaul, Matteo Frigo, Justin Mazzola Paluska, and Alexander (Sasha) Sandler, which describes the filesystem underlying the file storage service provided by Oracle's cloud infrastructure. It is our hope that you find these papers interesting and insightful.

Dahlia Malkhi and Dan Tsafrir

Program Co-Chairs

USENIX ATC 2019

(C) 2020 Copyright held by the owner/author(s).

1553-3077/2020/03-ART1

https://doi.org/10.1145/3383194 